\title{
Reintegrieren statt vorzeitig invalid-pensionieren
}

\author{
H. Schmidt
}

a Das ist eine Schätzung des Autors. Wir wissen zwar, wie viele Briefträger im Jahr von Hunden gebissen werden, wie hoch jedoch genau die Leistungen aller Beteiligten (Arbeitgeber/Privat- und Sozialversicherungen) sind, ist nicht bekannt.

b Case Management bezeichnet einen Prozess der Zusammenarbeit, in dem es um das Einschätzen, Planen, Umsetzen, Koordinieren und Überwachen der Aktivitäten aller Beteiligten zur qualitätsvollen und kostensparenden Erbringung einer individuellen Leistung geht, siehe dazu [2-6].

c Website der kanadischen Organisation NIDMAR www.nidmar.ca, Forschungsberichte aus Irland, im Auftrag der EU erstellt, www.wrc-research.ie/return/, vgl. auch $[7,8]$.

\section{Korrespondenz: \\ Hans Schmidt \\ Lic.oec./Rechtsanwalt \\ Bahnhofstrasse 10 \\ CH-8700 Küsnacht}

E-mail: h.schmidt@rehafirst.ch

\section{Das Problem}

Im Schatten der Diskussion der steigenden Gesundheitskosten versteckte sich bis vor kurzem ein anderes Problem: Die galoppierenden Invaliditätskosten. Zahlen gefällig? Anfang dieses Jahres wird die Invalidenversicherung (IV) an gegen 300000 Personen Renten für das vorzeitige gesundheitsbedingte Ausscheiden aus dem Erwerbsleben zahlen. Und Zehntausende von Gesuchstellern warten noch auf einen Entscheid.

Stolz weist man bei der IV im Rahmen der jüngst aufgeflammten «Scheininvaliden-Diskussion» darauf hin, dass ja 30\% der Gesuche abgelehnt würden, verschleiert dabei aber, dass es viele Gesuchsteller mehrmals versuchen, eine Rente zu erlangen. Nach jahrelangem Warten stehen am Schluss der jeweiligen Einzelschlacht um eine Invalidenrente gleich zwei Verlierer fest: das Versicherungssystem, das schliesslich fast immer zahlen muss, und der Klient selber: psychisch vom Kampf um die Existenz gezeichnet, der Arbeitsstelle beraubt, oft isoliert, aber mit Rente!

Dieser Entwicklung will man bei der Invalidenversicherung in nächster Zeit vor allem mit einer «strengeren» Haltung begegnen. Mit eigenen untersuchenden Ärzten will die IV die Hürde für die Rentenbezüger höher schrauben. Das Abwehrdispositiv wird nicht aufgehen: Eine höhere Hürde führt zu einem geänderten Verhalten auf seiten der häufig verzweifelten Rentenersuchenden. Diese werden angesichts einer existenziellen Angst noch kränker und verursachen in diesem pitoyablen Gesundheitszustand noch mehr Heilbehandlungskosten. Und wer bezahlt? Vorerst die Arbeitslosenkasse und die Sozialämter, bis dann vielleicht die Migrationsämter bei fürsorgeabhängig gewordenen Ausländern zur Familienausweisung schreitet. Spätestens dann bricht Panik und Verzweiflung beim Gesuchsteller aus, ein Gesundheitsschaden mit Krankheitswert wird spätestens jetzt zu Invaliditätsleistungen führen.

Folge: Die Invaliditätszahlen werden weiter steigen. Fast jeder vierte Mann bezieht vor Erreichen der AHV-Altersgrenze bereits eine
IV-Rente. Alleinerziehende Mütter werden weiter ein viermal höheres Invaliditätsrisiko zu tragen haben. Und nebst den jetzt 16 Milliarden Franken $^{\mathrm{a}}$ an jährlichen Renten und Taggeldern aus den verschiedenen Versicherungssystemen werden auch die indirekten Kosten (Produktivitätsverluste, Wiedereinstellungskosten, tiefere Steuereinnahmen) weiter in die Höhe klettern.

Und ein Reservoir von $15 \%$ der heutigen Mitarbeiter steht nicht allzu weit vom Eingangstor des Rentensystems. Eine Kündigung aus strukturellen Gründen, eine längere Arbeitslosigkeit macht sie zu «Kunden» (offizieller Jargon) oder besser gesagt zu Wartenden in einem passiven IV-System [1]. Denn nicht selten vergehen Jahre, bis es nach langem Formularesammeln seitens der IV und dem Warten des «Kunden» auf ein Gutachten zu einer Rente kommt.

\section{Der andere Weg}

Was es braucht ist mehr Zeit, mehr Verständnis, mehr Personal, um die Reintegration der von einem Gesundheitsschaden Betroffenen zu unterstützen. Wir brauchen nicht neue Strategien für die Rentenabwehr, was wir brauchen ist ein national abgestützter Wiedereingliederungsangriff. Mit Erhöhung der Zahl der «Polizisten» (die IV-Ärzte dürfen neu selber untersuchen), mit Erschwerung des Zugangs zum Rentensystem werden keine Arbeitsplätze geschaffen. Der alleinige Weg führt über eine allenfalls teilweise Reintegration am bereits bestehenden Arbeitsplatz. Wo dieser verloren ist braucht es viel Energie, Personal, Geld und Ideen, um neue, behindertengerechte Arbeitsplätze zu schaffen.

Das mag utopisch klingen, in vielen Ländern ist diese Idee bereits heute in Tausenden von Betrieben Realität geworden. In Australien, Kanada, den Niederlanden, in Irland und nun auch in Deutschland werden vermehrt externe oder interne Case Manager ${ }^{\mathrm{b}}$ damit beauftragt, eine gezielte Reintegrationspolitik von erkrankten und verunfallten Mitarbeitern zu betreiben. Es geht dabei um Disability Management, das vor allem in $\operatorname{Kanada}^{\mathrm{c}}$ weiterentwickelt worden ist. 
«Gemanagt» wird dabei nicht der «Fall», sondern die Schwierigkeiten, die bei der Reintegration auftreten können. Ziel ist es also, in unserem fraktionierten Sozialversicherungs- und Heilbehandlungssystem eine prozessorientierte Begleitung aufzuziehen (Fallführung aus einer Hand).

Gleich drei «natürliche Feinde» bedrohen diese Integration. Der Vorgesetzte: «Ich kann Sie erst wieder brauchen, wenn Sie ganz gesund sind!» Der Betroffene: «Ich will zuerst ganz gesund werden, Herr Doktor, ich mache alles, was Sie mir empfehlen!» Der Arzt: «In diesem Zustand lasse ich Sie nicht zur Arbeit gehen!» Dabei wäre es in vielen Fällen sehr wichtig, dass die Betroffenen den Kontakt zum Arbeitsplatz nicht verlieren. Wir wissen, je länger die Arbeitsunfähigkeit dauert, desto unwahrscheinlicher ist die Rückkehr an den Arbeitsplatz. Deshalb geht es vermehrt darum, auch kleine Arbeitsfähigkeiten (z.B. 10-30\%) möglichst bald am Arbeitsplatz selber umzusetzen. Diese möglichst frühe, sichere Rückkehr an den Arbeitsplatz ist Sache des Case Managers.

Werden wir es in der Schweiz schaffen, vermehrt dem Grundsatz nachzuleben: «Arbeit als Rehabilitation statt Rehabilitation vor Arbeit»? Die Versicherer unterstützen heutzutage solche Bemühungen, indem sie weiterhin vorerst die vollen Taggeldleistungen erbringen und nach einigen Wochen erst die ungefähre Leistungsfähigkeit zusammen mit dem Arbeitgeber und dem Betroffenen ermitteln.

\section{Geschäftsleitung muss hinter dem Programm stehen}

Dieses System kann nur funktionieren, wenn die Geschäftsleitung beschliesst: Bei uns gilt die Devise Arbeitsplatzsicherheit nach Unfall und Krankheit. Und dies aus der Erkenntnis heraus, dass die Kündigung erkrankter oder verunfallter Mitarbeiter nur für das Profitcenter «Betrieb» rentiert oder besser gesagt billiger kommt. Durch die Hintertüre werden entlassene geschwächte Mitarbeiter dem Profitcenter «Pensionskasse» oder der Krankentaggeldversicherung (die dann im nächsten Jahr bei steigenden Fallzahlen die Prämie wieder erhöht) belastet. Dabei könnte in vielen Fällen eine bestehende Restarbeitsfähigkeit am bisherigen oder an einem neuen Arbeitsplatz im Betrieb genutzt werden. Ein betrieblicher Case Manager kümmert sich nicht bloss um Anpassungen am bestehenden Arbeitsplatz, er sucht auch inner- oder ausserhalb des Betriebes nach neuen Einsatzmöglichkeiten. Pro
1000 Mitarbeiter rechnet man mit einem Case Manager, der realistischerweise 30 Personen gleichzeitig begleiten kann.

Zum Mentalitätswechsel gehört es auch, dass intern auch Reintegrationsarbeitsplätze gezielt geschaffen werden. Bisher ist in vielen Betrieben darüber kaum ein Gedanke verschwendet worden. Doch wenn alle Chefs sagen: «Wir haben keine geeigneten Arbeitsplätze für gesundheitlich eingeschränkte Personen, bei uns muss jeder $120 \%$ bringen», kommt es unweigerlich zu Stellenverlusten und damit auch zwangsläufig zu steigenden Invaliditätszahlen. Denn wer will schon einen angeschlagenen Stellenlosen wieder einstellen? Wir sollten vermeiden, dass dieser Weg über Arbeitslosigkeit, Hürdenlauf durch das Sozialversicherungssystem am Schluss auf einer Rutschbahn der Verzweiflung mit einer Rente endet. In unserer widersprüchlichen Welt der zerrissenen Sinnzusammenhänge wächst das Risiko des Scheiterns. Menschen wird gekündigt, sie finden keinen neuen Job, fallen aus ihren Rollen in das für viele unbequeme Netz der sozialen Sicherheit.

\section{Handeln sofort möglich}

Dieser neue Weg kann in der Schweiz sofort beschritten werden. Ein Warten darauf, bis die Invalidenversicherung auf dem Gebiet des Arbeitsplatzerhaltes wirklich einsatzfähig wird, ist unnötig. ${ }^{\mathrm{d}}$ Jedes Unternehmen kann theoretisch morgen eine solche Reintegrationspolitik durch die Geschäftsleitung beschliessen lassen. Wichtig dabei ist, dass auch die Versicherer und Gewerkschaften mit in die Pilotprojekte einbezogen werden. Mit konsequenter Einführung und Durchsetzung dieses Gedankens lassen sich nach internationalen Erfahrungen 20-50\% der Versicherungskosten einsparen. Pro eingesetztem Franken kommen fünf Franken zurück. Das zeigt das Beispiel einer kanadischen Papierfabrik mit 800 Mitarbeitern in Port Alberni/British Columbia. 1994 bezahlte diese Firma für Versicherungsprämien rund 4,7 Millionen Schweizer Franken. Nach Einführung eines Case-Management-Programms konnten die Versicherungskosten innerhalb von fünf Jahren auf 1,9 Millionen Franken gesenkt werden. Der Mitteleinsatz, um diese $4 \%$ Senkung der Lohnkosten zu erzielen, betrug knapp 500000 Franken, dagegen belief sich die Nettoersparnis auf 2,8 Millionen Franken. Eine klassische Win-win-Situation also...

Acht Grossbetriebe in der Schweiz erwägen momentan, das kanadische Modell in Pilotversuchen selbst zu erproben [9]. Sie schwimmen 
gegen den Strom in einer Arbeitswelt, die sonst immer rascher Nachschub für die Invalidenversicherung schafft.

\section{Case Management in aller Munde}

Die Methode des Case Managements findet in komplexen Problemlagen vermehrt Anwendung. Auch die Suva hat das Steuer ihres Schadenerledigungsdampfers herumgerissen. Über 40 Arbeitsgruppen haben einen tiefgreifenden Mentalitätswechsel vorbereitet. Die Suva will sich bei der Schadenerledigung in Zukunft auf komplexe Fälle mit mehr als vier Wochen Arbeitsunfähigkeit konzentrieren. Über die Zuständigkeitsgrenzen hinaus will die Suva Vorleistungen erbringen, rasch handeln, mit gegen 150 internen Case Managern aus einer Hand versuchen, Invalidität zu vermeiden oder $\mathrm{zu}$ reduzieren.

Zweites Beispiel: Die vier grossen Versicherer haben sich gemeinsam mit über 20 Anwälten, die Verunfallte vertreten, zur Activita Care Management, Uster [10], zusammengeschlossen. Ziel dieser allparteilichen, paritätisch geführten Organisation ist es, möglichst frühzeitig Fälle nach HWS-Verletzungen oder Hirnerschütterungen zugewiesen zu bekommen. Erste erfreuliche Erfahrungszahlen nach zwei Jahren liegen vor: Von den bisher abgeschlossenen Fällen waren $55 \%$ wieder voll- und $15 \%$ teilarbeitsfähig. $6 \%$ waren in einer Umschulung, 24\% waren nicht arbeitsfähig. Dies entspricht insgesamt einer unerwartet hohen Erfolgsquote. Eine Kundenumfrage der Activita, die gerade kürzlich durchgeführt worden ist, ergab eine sehr hohe Zufriedenheit, insbesondere der verunfallten Personen, aber auch der Ärzte und Therapeuten.

Die Methode des Case Managements eignet sich nicht nur zur Vermeidung von Langzeitinvalidität. Auch Krankenversicherer (im Tag- geldbereich wie auch bei komplexen Krankheitsbildern), Spitäler, Sozialämter usw. arbeiten vermehrt mit dieser Methode. Bereits gibt es zwei Ausbildungsmöglichkeiten und ein Netzwerk, das alle Interessierten unter einem Dach vereinigt. An der ersten Tagung im vergangen September nahmen mehr als 250 Personen teil [11].

\section{Literatur}

1 Schmidt H. Doppelt diskriminiert. Managed Care 2003;7:21-3.

2 Ewers M, Schaffer D. Case-Management in Theorie und Praxis. Bern: Huber; 2000.

3 Löcherbach P, Klug W, Remmel-Fassbender R, Wendt WR (Hrsg.). Case Management: Fall- und Systemsteuerung in Theorie und Praxis. Neuwied: Luchterhand; 2002.

4 Schmidt H, et al. Case Management zur Verhinderung von Langzeitinvalidität. Managed Care 2001;5:8-27. (Titel vergriffen, aber über Archiv im Internet unter www.fmc.ch herunterladbar).

5 Van Riet N, Wouters H. Case Management - Ein Lehr- und Arbeitsbuch über die Organisation und Koordination von Leistungen im Sozial- und Gesundheitswesen. Luzern: Interact; 2002.

6 Wendt WR. Case Management im Sozial- und Gesundheitswesen. Freiburg: Lambertus; 1999.

7 Bloch S, Prins R. Who Returns to Work \& Why? A Six Country Study on Work Incapacity \& Reintegration. New Brunswick/London: Transaction Publishers; 2000.

8 National Institute of Disability Management and Research. Strategies for Success, Disability Management in the Workplace. 1997. ISBN 0-9682480-0-4.

9 Hollenstein P. Invaliden Lohn zahlen und Geld sparen. NZZ am Sonntag, 2. November 2003, S. 27.

10 www.activita.ch.

11 www.netzwerk-cm.ch. 